\title{
SOME INVARIANT PROPERTIES ON SUMMABILITY DOMAINS
}

\author{
SHEN-YUE KUAN
}

\begin{abstract}
Let $A$ be an infinite matrix. Each $f \in c_{A}^{\prime}$ has a representation $f(x)=\alpha \lim _{A} x+t(A x)+r x$. The purpose of this short article is to answer the following problems raised by Wilansky. 1. Does $\alpha$ invariantly unique imply $\alpha^{\perp}$ invariant? 2. Does $A$ not-replaceable imply $\alpha^{\perp}$ invariant? 3 . Could a function $f \in c_{A}^{\prime}$ with $\alpha$ uniquely zero have a matrix representation?

4. Is the set of test functions invariant?
\end{abstract}

We assume that $A$ is a fixed matrix with convergent columns, i.e. $c_{A} \supset \varphi$, the finite sequences. Every $f \in c_{A}^{\prime}$ has a representation

$$
f(x)=\alpha \lim _{A} x+t(A x)+r x
$$

where $t \in l, r \in c_{A}^{\beta}, t(A x)=\sum_{n} t_{n}(A x)_{n}, r x=\Sigma_{k} r_{k} x_{k}$.

The representation (1) is far from unique for $f$ and we say $\alpha$ is unique for $A$ if all representations for $f$ have the same $\alpha$. It is easy to see that $\alpha$ is unique for one $f$ iff $\alpha$ is unique for all $f$.

$\alpha$ is said to be invariantly unique of $\alpha$ is unique for every $B$ with $c_{A}=c_{B}$. If $\alpha$ is invariantly unique, and $B$ is any matrix with $c_{A}=c_{B}$, and $f \in c_{A}^{\prime}$, we write $\alpha_{A}(f), \alpha_{B}(f)$ for the values of $\alpha$ when $f$ is expressed in the form (1) with respect to $A$ or $B$. Put $\alpha_{A}^{\perp}=\left\{f \in c_{A}^{\prime} ; \alpha_{A}(f)=0\right\}$ and similarly for $\alpha_{B}^{\perp}$. If $\alpha_{A}^{\perp}=\alpha_{B}^{\perp}$ for every $B$ with $c_{A}=c_{B}$, we say $\alpha^{\perp}$ is invariant.

The following problems were raised by Wilansky in [1].

1. Does $\alpha$ invariantly unique imply $\alpha^{\perp}$ invariant?

2. Does $A$ not-replaceable imply $\alpha^{\perp}$ invariant? (Here we assume $A$ is conversative.)

3. Could a function $f \in c_{A}^{\prime}$ with $\alpha$ uniquely zero have a matrix representation? I.e. there is a matrix $B$ with $c_{A}=c_{B}, \lim _{B}=f$.

It is known that if $f$ has a representation (1) with $\alpha \neq 0, f$ has a matrix representation. (See [3, Satz 5.3].) We observe that if $\alpha$ is not invariantly unique, there is a matrix $D$ with $c_{D}=c_{A}$ such that $f$ has a representation (1) (in $D$-form) with $\alpha \neq 0$. The above known result which we have just mentioned tells us that $f$ has a matrix representation. Thus it remains to consider Problem 3 in the case that $\alpha$ is invariantly unique.

Received by the editors August 30, 1976.

AMS (MOS) subject classifications (1970). Primary 40H05, 46A45.

Key words and phrases. Summability, nonreplaceable matrix, test function, matrix representation.

() American Mathematical Society 1977 
A function $f \in c_{A}^{\prime}$ is called a test function if $f=0$ on $\varphi$ and $\alpha=0$ in some representaton of $f$.

The next problem was raised by Wilansky in [2].

4. Is the set of test functions invariant?

All these problems can be solved by use of the following factorization theorem given in [2].

THEOREM 1. Let $A$ satisfy $c_{A} \supset \varphi$ and suppose that $\alpha$ is unique for $A$. Let $c_{B} \supset c_{A}$. Then there exist matrices $C, D$ such that (a) $B=C A+D$, (b) $\|C\|<\infty$, and for all $x \in c_{A}, t \in l, y=A x$ we have: (c) $t C \in l$, (d) (CA)x $=C(A x),(\mathrm{e}) t(D x)=(t D) x,(\mathrm{f}) t(C y)=(t C) y$.

The next four theorems answer the above four problems respectively.

THEOREM 2. If $\alpha$ is invariantly unique, then $\alpha^{\perp}$ is invariant.

Proof. Let $A, B$ be two matrices with $c_{A}=c_{B}$ and let $f \in \alpha_{B}^{\perp}$ and so $f$ can be written in the form $f(x)=t(B x)+r x$. Then, by Theorem 1 ,

$$
f(x)=t[(C A+D) x]+r x=(t C)(A x)+[(t D)+r] x .
$$

Since $t C \in l$ we have $f \in \alpha_{A}^{\perp}$. Hence $\alpha_{B}^{\perp} \subset \alpha_{A}^{\perp}$. Similarly we can prove $\alpha_{A}^{\perp} \subset \alpha_{B}^{\perp}$. Thus we conclude that $\alpha^{\perp}$ is invariant.

THEOREM 3. If $A$ is not-replaceable, then $\alpha^{\perp}$ is invariant.

Proof. Since the replaceability is an invariant property, then [1, Theorem 2.3] $\alpha$ is invariantly unique. Thus this theorem follows from Theorem 2 .

THEOREM 4. Let $f \in c_{B}^{\prime}$ with $\alpha_{B}(f)$ uniquely zero. If $\alpha$ is invariantly unique, then $f$ could not have a matrix representation.

Proof. Suppose there is a matrix $A$ with $c_{B}=c_{A}$ such that $f=\lim _{A}$. Then, by Theorem 1,

$$
\lim _{A} x=t(B x)+r x=t[(C A+D) x]+r x=(t C)(A x)+[t D+r] x .
$$

But this would imply $0=\lim _{A} x+t^{\prime}(A x)+r^{\prime} x$ where $t^{\prime}=-t C, r^{\prime}=t D+$ $r$. This contradicts that $\alpha$ is invariantly unique.

THEOREM 5. The set of test functions is invariant.

Proof. Let $A, B$ be two matrices with $c_{A}=c_{B}$. Let $T_{A}$ be the set of all test functions with respect to $A$ and similarly for $T_{B}$. If $f \in T_{B}$ we consider the following two cases:

CASE $1 . \alpha$ is not unique for $A$. We can write $f$ in two different representations

$$
f(x)=\alpha_{1} \lim _{A} x+t_{1}(A x)+r_{1} x=\alpha_{2} \lim _{A} x+t_{2}(A x)+r_{2} x .
$$

Let $\lambda=\alpha_{2} /\left(\alpha_{2}-\alpha_{1}\right)$. Then $f=\lambda f+(1-\lambda) f$ expresses $f$ with $\alpha=0$.

CASE 2. $\alpha$ is unique for $A$. Since $f \in T_{B}, f$ can be expressed in the form $f(x)=t(B x)+r x$. Then, by Theorem 1 , 


$$
f(x)=t[(C A+D) x]+r x=(t C)(A x)+[(t D+r)] x
$$

with $t C \in l$.

Thus $f$ always has a representation (1) with $\alpha=0$ with respect to $A$. Of course $f=0$ on $\varphi$ in $c_{A}$ since $f \in T_{B}$ and $c_{A}=c_{B}$. So $f \in T_{A}$ and hence $T_{B} \subset T_{A}$. Similarly we can prove $T_{A} \subset T_{B}$. This completes the proof.

\section{REFERENCES}

1. M. S. Macphail and A. Wilansky, Linear functionals and summability invariants, Canad. Math. Bull. 17 (1974), 233-242. MR 50 \# 13973.

2. A. Wilansky, On the $\mu$ property of FK spaces, Comment. Math., Special Volume dedicated to W. Orlicz on the occasion of his 75th birthday, 1978 (to appear).

3. K. Zeller, Allegemeine Eigenschaften von Limitierungsverfahren, Math. Z. 53 (1951), 463-487. MR 12, 604.

Department of Mathematics, National Central University, Chung Li, Taiwan, RepubLic of ChINA 